\title{
The 4-metre gait speed in COPD: responsiveness and minimal clinically important difference
}

\author{
Samantha S.C. Kon ${ }^{1,3}$, Jane L. Canavan ${ }^{1,3}$, Claire M. Nolan ${ }^{1,2}$, Amy L. Clark², \\ Sarah E. Jones', Paul Cullinan', Michael I. Polkey and William D-C. Man ${ }^{1,2}$
}

Affiliations: ${ }^{1}$ NIHR Respiratory Biomedical Research Unit, Royal Brompton and Harefield NHS Foundation Trust and Imperial College, London and ${ }^{2}$ Harefield Pulmonary Rehabilitation Unit, Royal Brompton and Harefield NHS Foundation Trust, London, UK. ${ }^{3}$ These authors contributed equally.

Correspondence: S.S.C. Kon, Dept of Respiratory Medicine, Harefield Hospital, Hill End Road, Harefield, UB9 6JH, UK. E-mail: s.konarbht.nhs.uk

ABSTRACT Usual gait speed is a consistent predictor of adverse outcomes in community-dwelling elderly people. The reliability and validity of the $4-\mathrm{m}$ gait speed (4MGS) has recently been demonstrated in patients with chronic obstructive pulmonary disease (COPD). The aims of this study were to assess the responsiveness of the $4 \mathrm{MGS}$ and to estimate the minimal clinically important difference (MCID).

In 301 COPD patients, 4MGS and incremental shuttle walk (ISW) were measured before and after pulmonary rehabilitation. 4MGS and ISW were also measured at baseline and 1 year later in a separate cohort of 162 COPD patients. The MCID of 4MGS was estimated using distribution and anchor-based methods.

4MGS improved significantly with pulmonary rehabilitation (mean change $0.08 \mathrm{~m} \cdot \mathrm{s}^{-1}, \mathrm{p}<0.001$ ). The minimal detectable change at $95 \%$ confidence was $0.11 \mathrm{~m} \cdot \mathrm{s}^{-1}$. The MCID was estimated at $0.11 \mathrm{~m} \cdot \mathrm{s}^{-1}$ (anchored against ISW) and $0.08 \mathrm{~m} \cdot \mathrm{s}^{-1}$ (anchored against self-reported improvement). The effect size for 4MGS was greatest in frail individuals. After 12 months, mean $4 \mathrm{MGS}$ declined by $0.04 \mathrm{~m} \cdot \mathrm{s}^{-1}$. When anchored against a decline of more than the MCID for ISW, change in 4MGS was $-0.11 \mathrm{~m} \cdot \mathrm{s}^{-1}$.

The 4MGS is responsive to pulmonary rehabilitation and longitudinal change in COPD, and has potential as a simple functional assessment tool in COPD. The 4MGS may be particularly useful in frail individuals with COPD.

@ERSpublications

4-m gait speed is responsive to pulmonary rehabilitation and longitudinal change with an estimated MCID of $0.11 \mathrm{~m} / \mathrm{s}$ http://ow.ly/pXdmQ

This article has supplementary material available from www.erj.ersjournals.com

Received: May 232013 | Accepted after revision: Sept 272013 | First published online: Oct 312013

Support statement: S.S.C. Kon is supported by the Medical Research Council (MRC). J.L. Canavan and S.E. Jones are supported by the National Institute for Health Research (NIHR) Respiratory Biomedical Research Unit at the Royal Brompton and Harefield NHS Foundation Trust and Imperial College London. W.D-C. Man is supported by an NIHR Clinician Scientist Award, an MRC New Investigator Research Grant and an NIHR Clinical Trials Fellowship. This project was undertaken at the NIHR Respiratory Biomedical Research Unit at the Royal Brompton and Harefield NHS Foundation Trust and Imperial College London. M.I. Polkey's salary is part funded by the Biomedical Research Unit. The views expressed in this publication are those of the authors and not necessarily those of the NHS, the NIHR or the Department of Health.

Conflict of interest: None declared.

Copyright @ERS 2014 


\section{Introduction}

In autonomous community-dwelling older people, usual gait speed has been shown to be a consistent risk factor for disability, nursing home admission and falls, as well as cardiovascular and all-cause mortality $[1,2]$. Gait speed is also an established marker of exercise performance in the elderly [1], correlates with other wellrecognised functional performance measures, such as 6-min walk distance [3], and has been shown to be a responsive measure of physical performance in patients undergoing short-term rehabilitation after acute hospital admission, hip fracture and stroke [4-6]. Expert consensus groups have recently identified gait speed as the most suitable functional test for pharmacological trials in frail older adults [7].

In chronic obstructive pulmonary disease (COPD), several field tests of exercise performance exist, including the 6-min walk distance, the incremental shuttle walk (ISW) and the endurance shuttle walk. These are well validated and provide useful information on the impact of disease on patient functioning and prognosis [8,9], and provide a way of assessing the effects of interventions [10]. However, limitations such as space, time and learning effect limit their widespread clinical use. We recently described the usual gait speed over $4 \mathrm{~m}$ in patients with COPD, and demonstrated excellent test-retest and interobserver reliability. Furthermore, the 4-m gait speed (4MGS) is strongly correlated with the ISW in stable outpatients with COPD [11]. As the 4MGS test takes $<2$ min to perform, and requires only a stopwatch and a short course, it may be a feasible outcome tool for patients with COPD in most clinical settings, including the home. However, the clinical value of the $4 \mathrm{MGS}$ as an outcome tool is dependent on its ability to detect change with time and intervention.

Therefore, the aims of this study were to assess the responsiveness of the 4MGS to intervention (pulmonary rehabilitation) and longitudinal change with time in stable outpatients with COPD. An additional aim was to estimate the minimal clinically important difference (MCID) for the 4MGS in COPD. We hypothesised that change in 4MGS would correlate significantly with change in ISW and be responsive to pulmonary rehabilitation, particularly in frail individuals.

\section{Methods}

\section{Participants}

Participants were recruited from pulmonary rehabilitation or outpatient respiratory clinics at Harefield Hospital, London, UK. Stable patients diagnosed with COPD according to the Global Initiative for Chronic Obstructive Lung Disease guidelines were recruited. Patients with unstable cardiac disease, recent exacerbation (within preceding 4 weeks), inability to walk $5 \mathrm{~m}$ or predominant neurological limitation to walking (e.g. significant hemiplegia) were excluded. All participants gave informed consent and the study was approved by the West London and the London-Camberwell St Giles Research Ethics Committees $(11 / \mathrm{H} 0707 / 2$ and $11 / \mathrm{LO} / 1780)$.

\section{Measurement of 4 MGS}

4MGS testing was performed as described previously [11]. Briefly, a 4-m flat, unobstructed course was identified and marked out with tape. Patients were asked to walk at their usual speed and a stopwatch was used to record the time taken to complete the 4-m course. The 4MGS test was repeated without rest and the faster of the two times recorded. The fastest time was used to calculate the 4MGS, expressed as metres per second. Participants were allowed to use their normal walking aids (e.g. cane or frame) and oxygen if required.

\section{Response to pulmonary rehabilitation}

430 patients with COPD referred for a multidisciplinary outpatient pulmonary rehabilitation programme between March 2011 and September 2012 participated in the study. The programme consisted of supervised exercise and education sessions twice a week for 8 weeks. Participants were also encouraged to perform unsupervised exercise at home.

The 4MGS was measured before and after pulmonary rehabilitation. In addition, the ISW [8], the Medical Research Council (MRC) dyspnoea scale [12], the COPD Assessment Test (CAT) [13], the Chronic Respiratory Questionnaire (CRQ) [14] and the St George's Respiratory Questionnaire (SGRQ) [15] were measured before and after pulmonary rehabilitation by assessors blinded to 4MGS results. A five-point Likert scale was used to assess patient self-reported response to pulmonary rehabilitation ranging from 1, which represented "feeling much better", to 5, which represented "feeling much worse" after pulmonary rehabilitation. Comorbidities were recorded as the Charlson Index [16] and the COPD-specific comorbidity test (COTE) index [17]. Response to pulmonary rehabilitation was calculated for the whole cohort.

Anchor- and distribution-based approaches were used to estimate the MCID of the 4MGS by calculating the mean change in $4 \mathrm{MGS}$ in those reporting feeling better after pulmonary rehabilitation ( 2 on the Likert 
scale) and in those achieving the known MCID for the ISW ( $>47.5 \mathrm{~m}$ improvement) [8]. The distributionbased approach used the calculated minimal detectable change at 95\% confidence (MDC95), the amount of change required to exceed what would be expected from measurement variability $[4,5]$.

As 4MGS is commonly used to screen for frail individuals [18], planned subgroup analysis included the change in 4MGS and ISW following pulmonary rehabiliation stratified according to baseline 4MGS.

\section{Longitudinal change in the 4 MGS}

200 patients with COPD were recruited from respiratory outpatient clinics at Harefield Hospital. 4MGS, ISW, CAT, SGRQ and spirometry were measured at baseline and at 12 months. MCID was estimated using an anchor-based approach by calculating the mean change in 4MGS over 12 month in those individuals showing a reduction in the ISW of $>47.5 \mathrm{~m}$ [8].

\section{Data analysis}

Data analyses and graphical presentations were performed using GraphPad Prism 5 (GraphPad Software, San Diego, CA, USA) or SPSS version 21 (IBM, Armonk, NY, USA). Paired t-tests or nonparametric equivalent were used to compare outcomes before and after PR and between baseline and 1 year. Correlation between change in 4MGS and change in other outcome measures was assessed using Pearson's correlation or Spearman's rank correlation. Substantially skewed data were log-transformed before analysis to calculate effect sizes. Cohen's d was calculated to estimate effect size using the mean difference and standard deviation before and after pulmonary rehabilitation; this allowed an assessment of the magnitude of change with pulmonary rehabilitation. MCID was assessed using both distribution- and anchor-based approaches, as described. A p-value $<0.05$ was considered significant.

\section{Results}

Response to pulmonary rehabilitation

Of the 430 patients referred for pulmonary rehabilitation, 120 were deemed non-completers as they failed to attend $\geqslant 50 \%$ of supervised sessions. Nine patients completed, but failed to attend assessment postrehabilitation; hence, data from 301 (70\%) participants (165 male and 136 female) were available for analysis. Baseline characteristics are presented as median (interquartile range): age 71 (64-76) years, forced expiratory volume in $1 \mathrm{~s}$ (FEV1) 49 (32-63)\% predicted, body mass index $26.7(23.4-30.9) \mathrm{kg} \cdot \mathrm{m}^{-2}$, Charlson index $1(1-2)$ and COTE index $0(0-1)$. Table 1 shows the changes in 4MGS, ISW, MRC, CAT, CRQ and SGRQ following pulmonary rehabilitation. There was no significant correlation between change in $4 \mathrm{MGS}$ and number of pulmonary rehabilitation sessions attended or baseline 4MGS. A significant increase in mean 4MGS with pulmonary rehabilitation $\left(0.08 \mathrm{~m} \cdot \mathrm{s}^{-1}\right)$ was demonstrated, which correlated significantly with change in ISW $(r=0.26 ; \mathrm{p}<0.001)$, and change in SGRQ, CRQ and CAT $(r=-0.18$, $\mathrm{r}=0.26$ and $-0.16 ; \mathrm{p}<0.01$ ). The effect size with PR for both the 4MGS and ISW in the whole cohort was 0.4.

Changes in 4MGS and ISW with pulmonary rehabilitation stratified according to baseline 4MGS (arranged into quartiles) are shown in figure 1. For patients in the lowest quartile for baseline 4MGS $\left(<0.77 \mathrm{~m} \cdot \mathrm{s}^{-1}\right)$, the mean change in 4MGS with pulmonary rehabiliation was $0.11 \mathrm{~m} \cdot \mathrm{s}^{-1}$ and the mean change in ISW was $61 \mathrm{~m}$. For this subgroup, effect size for 4MGS and ISW were 1.0 and 0.6 , respectively. With pulmonary rehabiliation, patients in the highest quartile for baseline 4MGS $\left(\geqslant 1.05 \mathrm{~m} \cdot \mathrm{s}^{-1}\right)$ improved mean 4MGS by $0.02 \mathrm{~m} \cdot \mathrm{s}^{-1}$ and mean ISW by $53 \mathrm{~m}$, corresponding to an effect size of 0.2 and 0.4 , respectively.

\section{Longitudinal change in 4 MGS}

Of 200 patients who consented for the study, 38 did not return for 12-month follow-up (12 died, six moved out of the area, 14 declined and six were no longer contactable). The remaining 162 patients ( 89 male and 73 female) had a median age of 70 (64-76) years. Table 2 shows changes in 4MGS, ISW, MRC, CAT, SGRQ and FEV1 \% pred from baseline to 12 months. There was a significant reduction in mean change of the 4MGS $\left(-0.04 \mathrm{~m} \cdot \mathrm{s}^{-1}\right)$ and median change in ISW $(-30 \mathrm{~m})$ over 12 months. FEV1\% pred did not change significantly within the same time period. There was a significant positive correlation between change in 4MGS and change in ISW ( $\mathrm{r}=0.45 ; \mathrm{p}<0.001)$ (fig. 2), and significant negative correlations with CAT and SGRQ over time $(\mathrm{r}=-0.26$ and $\mathrm{r}=-0.34 ; \mathrm{p}<0.001)$.

\section{Minimal clinically important difference}

The MDC95 was $0.11 \mathrm{~m} \cdot \mathrm{s}^{-1}$. Using an anchor-based approach, the mean (95\% CI) change in 4MGS in those achieving the MCID in the ISW following pulmonary rehabilitation was $0.11(0.09-0.13) \mathrm{m} \cdot \mathrm{s}^{-1}$. For those "feeling better" after pulmonary rehabilitation, the mean change in 4MGS was $0.08(0.05-0.10) \mathrm{m} \cdot \mathrm{s}^{-1}$. Using the most conservative estimate for the MCID $\left(0.11 \mathrm{~m} \cdot \mathrm{s}^{-1}\right), 40 \%$ of all patients undergoing pulmonary improved their $4 \mathrm{MGS}$ by the MCID, compared with 51\% achieving the MCID in ISW. In the frail group, 
TABLE 1. Response to pulmonary rehabilitation (PR)

\begin{tabular}{|c|c|c|c|c|}
\hline Outcome & Pre-PR & Post-PR & Change with PR & p-value \\
\hline 4MGS $\mathrm{m} \cdot \mathrm{s}^{-1}$ & $0.90 \pm 0.22$ & $0.98 \pm 0.22$ & $0.08 \pm 0.13$ & $<0.001$ \\
\hline ISW m & $200(100-330)$ & $250(155-380)$ & $50(10-95)$ & $<0.001$ \\
\hline ISW \% predicted & $33(17-53)$ & $43(26-66)$ & $8(2-16)$ & $<0.001$ \\
\hline MRC dyspnoea score & $3(2-4)$ & $3(2-3)$ & $-1(-1-0)$ & $<0.001$ \\
\hline CAT score & $22(16-27)$ & $19(13-24)$ & $-2(-7-1)$ & $<0.001$ \\
\hline \multicolumn{5}{|l|}{ SGRQ score } \\
\hline Symptoms domain & $67.0(51.5-79.0)$ & $61.1(48.4-76.6)$ & $-3.1(-13.1-3.3)$ & $<0.001$ \\
\hline Activities domain & $72.3(59.5-85.5)$ & $72.3(53.5-85.8)$ & $-0.2(-12.7-6.6)$ & $<0.01$ \\
\hline Impacts domain & $36.0(24.0-48.0)$ & $30.4(20.7-44.0)$ & $-4.0(-12.8-2.2)$ & $<0.001$ \\
\hline Total score & $51.4(41.2-62.7)$ & $48.1(35.4-58.8)$ & $-3.8(-11.0-2.5)$ & $<0.001$ \\
\hline CRQ total score & 73 (59-92) & $91(74-108)$ & $15(4-27)$ & $<0.001$ \\
\hline
\end{tabular}

Data are presented as mean \pm SD or median (interquartile range), unless otherwise stated. $n=301$. 4MGS: 4-m gait speed; ISW: incremental shuttle walk; MRC: Medical Research Council; CAT: COPD Assessment Test; SGRQ: St George's Respiratory Questionnaire; CRQ: Chronic Respiratory Questionnaire.

55\% increased their 4MGS by the MCID compared to 47\% achieving the MCID in ISW. In the longitudinal study, patients with a $>47.5-\mathrm{m}$ decline in ISW over 12 months showed a mean change in $4 \mathrm{MGS}$ of $-0.11 \mathrm{~m} \cdot \mathrm{s}^{-1}$.

\section{Discussion}

This study demonstrates that 4MGS is responsive to intervention and declines with time in patients with COPD. Changes in $4 \mathrm{MGS}$ with pulmonary rehabilitation or with time correlated significantly with changes in ISW and health-related quality of life. Anchor- and distribution-based methods were consistent in estimating the MCID of the 4MGS to be between 0.08 and $0.11 \mathrm{~m} \cdot \mathrm{s}^{-1}$ in patients with COPD. Before discussing these points further, the current literature is briefly reviewed.

\section{Previous studies}

Recovery of function or walking ability is a common goal after rehabilitation. Several previous studies have utilised the usual gait speed as an outcome measure, particularly in patients who have received rehabilitation or exercise interventions after recent hospitalisation, hip fracture, spinal cord injury or stroke [4-6, 19, 20]. Palombaro et al. [5] pooled data from three studies on 92 patients who had gait speed measured before and after an exercise trial post-hip fracture, and demonstrated a MDC95 of $0.08 \mathrm{~m} \cdot \mathrm{s}^{-1}$. Based on clinical expert opinion and anchoring against the Timed "Up \& Go" Test [5], the MCID was estimated at $0.10 \mathrm{~m} \cdot \mathrm{s}^{-1}$. BARTHULY et al. [4] studied elderly patients undergoing short-term rehabilitation
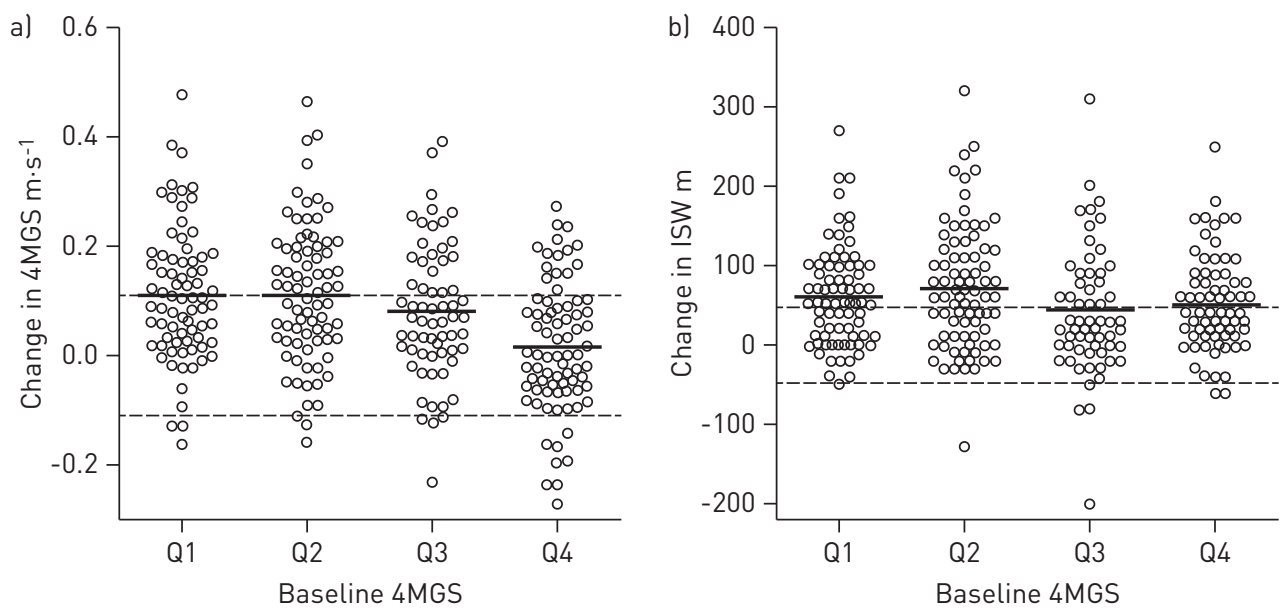

FIGURE 1 Scatterplot showing individual values and mean (solid line) change in a) 4-m gait speed (4MGS) and b) incremental shuttle walk (ISW) with pulmonary rehabilitation stratified according to baseline 4MGS quartiles (Q1: $<0.77 \mathrm{~m} \cdot \mathrm{s}^{-1}$; Q2: 0.77-0.91 m $\cdot \mathrm{s}^{-1}$; Q3: 0.92-1.04 m. $\mathrm{s}^{-1}$; Q4: $>1.05 \mathrm{~m} \cdot \mathrm{s}^{-1}$ ). Dashed lines represent an improvement and decline by the minimal clinically important difference $\left(0.11 \mathrm{~m} \cdot \mathrm{s}^{-1}\right.$ for $4 \mathrm{MGS} ; 47.5 \mathrm{~m}$ for ISW). 
TABLE 2 Longitudinal change over 1 year

\begin{tabular}{|c|c|c|c|}
\hline Outcome & Baseline & Change over 1 year & p-value \\
\hline Body mass index $\mathrm{kg} \cdot \mathrm{m}^{-2}$ & $26.4(24.1-31.6)$ & $0.2(-0.5-1.2)$ & $<0.001$ \\
\hline 4MGS $\mathrm{m} \cdot \mathrm{s}^{-1}$ & $0.90 \pm 0.26)$ & $-0.04 \pm 0.16]$ & $<0.001$ \\
\hline ISW m & $220(100-340)$ & $-30(-\overline{70}-20)$ & $<0.001$ \\
\hline ISW \% predicted & $40(20-57)$ & $-5(-10-3)$ & $<0.001$ \\
\hline MRC dyspnoea score & $3(2-4)$ & $0(-1-1)$ & 0.096 \\
\hline CAT score & $22(16,27)$ & $1(-4,4)$ & 0.745 \\
\hline \multicolumn{4}{|l|}{ SGRQ score } \\
\hline Symptoms domain & $61.5(21.8)$ & $-2.2(18.3)$ & 0.191 \\
\hline Activities domain & $68.7(23.0)$ & $-0.5(15.7)$ & 0.710 \\
\hline Impacts domain & $36.7(19.2)$ & $-2.4(13.6)$ & 0.057 \\
\hline Total score & $50.6(18.4)$ & $-1.7(11.7)$ & 0.110 \\
\hline FEV1 \% predicted & $46.1(19.9)$ & $0.8(31.6)$ & 0.840 \\
\hline
\end{tabular}

Data expressed as median (interquartile range) or mean \pm SD, unless otherwise stated. $n=162$. 4MGS: 4-m gait speed; ISW: incremental shuttle walk; MRC: Medical Research Council; CAT: COPD Assessment Test; SGRQ: St George's Respiratory Questionnaire; FEV1: forced expiratory volume in $1 \mathrm{~s}$.

following an acute hospital admission. Using an anchor-based method (against four different comparators), the MCID for the usual gait speed was estimated between 0.10 and $0.18 \mathrm{~m} \cdot \mathrm{s}^{-1}$ with an associated MDC95 of $0.13 \mathrm{~m} \cdot \mathrm{s}^{-1}$.

The usual gait speed has also been demonstrated to be responsive to natural recovery after stroke [5, 21], and to the natural ageing process in community-dwelling older persons $[22,23]$. In an observational study of older adults, PERERA et al. [22] estimated the smallest real difference in gait speed decline to be $0.05 \mathrm{~m} \cdot \mathrm{s}^{-1}$ and the MCID to be between 0.05 and $0.12 \mathrm{~m} \cdot \mathrm{s}^{-1}$, while ONDER et al. [23] demonstrated a 5.2\% change in gait speed from baseline to 1 year in moderately to severely disabled females.

Our study is the first to examine the responsiveness of the 4MGS in a COPD-specific population. A strength of the study was that we used two "interventions" to examine responsiveness: firstly, we were able to prospectively show significant improvement in the 4MGS with pulmonary rehabilitation; and secondly, we were also able to demonstrate a statistically significant decline in the 4MGS with time (>12 months). Changes in 4MGS correlated with changes in ISW, an established field walking measure of exercise capacity, providing further evidence of the longitudinal validity of the 4MGS in COPD. The magnitude of change was

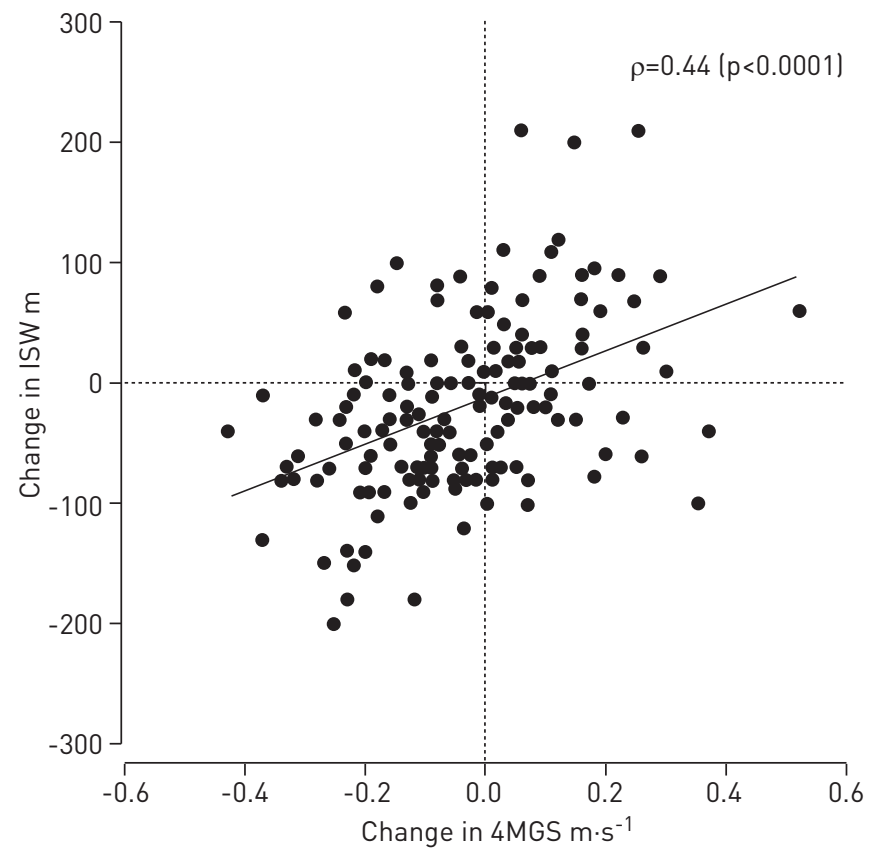

FIGURE 2 Relationship between change in 4-m gait speed (4MGS) and change in incremental shuttle walk (ISW) over 1 year. 
similar to those seen in other elderly populations [5,22]. Another strength of the study was that we used both multiple anchor- and distribution-based methods to calculate the MCID of the 4MGS, allowing us greater confidence in the validity of our estimate of the MCID. As the relationships between change in 4MGS and change in ISW and Likert scale in response to pulmonary rehabilitation were modest $(\mathrm{r}=0.26$ and $r=0.20$, respectively), caution is required in interpreting the results, but reassuringly, the estimates were consistent $\left(0.08-0.11 \mathrm{~m} \cdot \mathrm{s}^{-1}\right)$, similar to values obtained with longitudinal decline and distribution-based approach, and similar to MCID estimates of the 4MGS in other populations [5, 22]. This lends greater weight to the inferences drawn from our data.

\section{Limitations of the study}

There are a number of limitations to determining the MCID, which are not unique to our study. Distribution-based approaches compare the change in the outcome to some measure of variability, but do not address the clinical importance to the patient. However, distribution-based methods are a well-accepted approach to estimating clinically important differences in outcome measures [24]. In contrast, anchorbased methods do take into account patient experience, but require a suitable external criterion that correlates significantly with the outcome measure of interest. Investigators have suggested an arbitrary minimum r-value of between 0.3 and 0.5 [25], although lesser degrees of association are needed to generate convincing inferences when multiple anchors are used (as in our study) [26]. Furthermore, our demonstration of $\mathrm{r}=0.45$ between ISW change and $4 \mathrm{MGS}$ change with time is in line with these arbitrary parameters [27], and in line with studies used to determine the MCID of the 6-min walk distance in patients with COPD [28]. For a multisystem outcome variable such as 4MGS, it is unlikely that a single clinical anchor could capture the same construct [24], hence our decision to use multiple anchors in different clinical situations with corroboration from a distribution-based approach.

Our study also highlighted a limitation associated with the 4MGS, namely a ceiling effect. This is likely to explain the slightly lower $r$-value $(r=0.26)$ seen between change in 4MGS and change in ISW with pulmonary rehabilitation. Although this does not affect the potential utility of the $4 \mathrm{MGS}$ as a simple screening tool for exercise intolerance [11] or as an outcome measure in frail individuals, the 4MGS may be less useful when testing the effects of intervention in high functioning patients. Figure 1 highlights the fact that the 4MGS was less responsive to intervention in those with better 4MGS at baseline, and for patients in the highest quartile, the effect size was small $(\mathrm{d}=0.2)$. This has been well described in the gerontology literature [29]. Conversely, our data also suggest that the 4MGS was particularly responsive in individuals with the lowest baseline 4MGS. A response effect size of 1.0 was seen in those within the lowest quartile for baseline 4MGS $\left(<0.77 \mathrm{~m} \cdot \mathrm{s}^{-1}\right)$; this corresponds closely to the cut-off of $0.80 \mathrm{~m} \cdot \mathrm{s}^{-1}$ that is commonly used in gerontology to identify frail individuals [1]. Although it is possible these findings could be secondary to "regression to the mean", it is noticeable that ISW response to pulmonary rehabiliation was similar across the 4MGS quartiles. No relationship was observed between baseline gait speed and change in gait speed with pulmonary rehabilitation, suggesting that 4MGS change is not linearly dependent on baseline 4MGS value.

Certainly, expert consensus groups have identified usual gait speed as the most suitable functional outcome measure for pharmacological trials in frail older adults [7]. In patients with COPD, the 4MGS may therefore have a particular role in the assessment of poorly functioning or frail individuals, for whom longer (both in time and distance) tests of exercise capacity are more difficult or impractical. Typical scenarios might be the critical care setting, hospitalised in-patients, those recovering from exacerbation or housebound patients. Future prospective studies in different populations are needed to confirm this hypothesis.

A further limitation to our study is that patients lost to follow-up or those who did not complete pulmonary rehabilitation were excluded from the final analysis. Although the completion rate of $70 \%$ is in line with, if not better than, rates described from other UK services, it is conceivable that the exclusion of the non-completers may have biased estimates of responsiveness of the 4MGS. The non-completers did show slower baseline 4MGS than the completers, despite similar lung function parameters (table S1 in the online supplementary material).

\section{Gait speed as an outcome measure in COPD}

The test-retest and interobserver reliability and concurrent validity of the 4MGS in patients with COPD have recently been reported [11]. The current study extends these observations by demonstrating responsiveness of the 4MGS and proposing a clinically meaningful change, further pre-requisites for an outcome tool. We propose that the $4 \mathrm{MGS}$ has potential as a simple assessment tool in COPD.

A significant advantage of the 4MGS is simplicity. Assessment time is typically $1-2 \mathrm{~min}$ and the test requires only a stopwatch. The 4MGS is also readily acceptable to patients as walking is a familiar activity, the test is easy to understand and the short distance may relieve any anxieties associated with having to complete a 
maximal or near-maximal test. Furthermore, it requires little space, which means the 4MGS could be adopted in most clinical environments. In comparison, well-established field walking tests in COPD, such as the 6-min walk or the shuttle walk, are associated with learning effect and need to be repeated after adequate rest (typically, a second test is performed on a separate day or at least after an adequate rest, e.g. $30 \mathrm{~min}$ ). Space can also limit their widespread use in some clinical environments. For example, the 6-min walk requires a $30-\mathrm{m}$ course. Some investigators would also argue that the 4MGS may be more indicative of activities of daily living. In a recent study, SHIMADA et al. [30] compared several physical performance tests to predict the need for help with personal care in 10351 adults aged $\geqslant 65$ years. Usual gait speed was more strongly correlated with the demand for personal care than other performance measures, with an odds ratio of 5.9 and an area under curve of $0.92-0.94$.

In summary, our study demonstrates that the 4MGS improves with pulmonary rehabilitation and declines with time in COPD, with an estimated MCID of $0.11 \mathrm{~m} \cdot \mathrm{s}^{-1}$. The 4MGS is a simple assessment tool that shows potential as a quick, practical functional outcome measure in patients with COPD that is suitable for use in most healthcare settings. The 4MGS may be particularly useful in frail individuals with COPD.

\section{Acknowledgements}

The authors would like to acknowledge the Harefield Pulmonary Rehabilitation team (D. Kaliaraju, M. Peasey, D. Dilaver, M. Curtis, D. Toms, E. Bone, M. Ng and K. Ingram) for their assistance in collecting the data.

\section{References}

1 Abellan van Kan G, Rolland Y, Andrieu S, et al. Gait speed at usual pace as a predictor of adverse outcomes in community-dwelling older people an International Academy on Nutrition and Aging (IANA) Task Force. J Nutr Health Aging 2009; 13: 881-889.

Studenski S, Perera S, Patel K, et al. Gait speed and survival in older adults. JAMA 2011; 305: 50-58.

Steffen TM, Hacker TA, Mollinger L. Age- and gender-related test performance in community-dwelling elderly people: six-minute walk test, Berg balance scale, Timed Up \& Go Test, and gait speeds. Phys Ther 2002; 82: 128-137. Barthuly AM, Bohannon RW, Gorack W. Gait speed is a responsive measure of physical performance for patients undergoing short-term rehabilitation. Gait Posture 2012; 36: 61-64.

5 Palombaro KM, Craik RL, Mangione KK, et al. Determining meaningful changes in gait speed after hip fracture. Phys Ther 2006; 86: 809-816.

6 Tilson JK, Sullivan KJ, Cen SY, et al. Meaningful gait speed improvement during the first 60 days poststroke: minimal clinically important difference. Phys Ther 2010; 90: 196-208.

7 Working Group on Functional Outcome Measures for Clinical Trials. Functional outcomes for clinical trials in frail older persons: time to be moving. J Gerontol A Biol Sci Med Sci 2008; 63: 160-164.

8 Singh SJ, Jones PW, Evans R, et al. Minimum clinically important improvement for the incremental shuttle walking test. Thorax 2008; 63: 775-777.

9 Polkey MI, Spruit MA, Edwards LD, et al. Six-minute-walk test in chronic obstructive pulmonary disease: minimal clinically important difference for death or hospitalization. Am J Respir Crit Care Med 2013; 187: 382-386.

10 Lacasse Y, Goldstein R, Lasserson TJ, et al. Pulmonary rehabilitation for chronic obstructive pulmonary disease. Cochrane Database Syst Rev 2006; 4: CD003793.

11 Kon SS, Patel MS, Canavan JL, et al. Reliability and validity of 4-metre gait speed in COPD. Eur Respir J 2013; 42: 333-340.

12 Fletcher CM, Elmes PC, Fairbairn AS, et al. The significance of respiratory symptoms and the diagnosis of chronic bronchitis in a working population. Br Med J 1959; 2: 257-266.

13 Dodd JW, Marns PL, Clark AL, et al. The COPD Assessment Test (CAT): short- and medium-term response to pulmonary rehabilitation. COPD 2012; 9: 390-394.

14 Williams JE, Singh SJ, Sewell L, et al. Development of a self-reported Chronic Respiratory Questionnaire (CRQSR). Thorax 2001; 56: 954-959.

15 Jones PW, Quirk FH, Baveystock CM. The St George’s Respiratory Questionnaire. Respir Med 1991; 85: Suppl. B, 25-31.

16 Charlson ME, Pompei P, Ales KL, et al. A new method of classifying prognostic comorbidity in longitudinal studies: development and validation. J Chronic Dis 1987; 40: 373-383.

17 Divo M, Cote C, de Torres JP, et al. Comorbidities and risk of mortality in patients with chronic obstructive pulmonary disease. Am J Respir Crit Care Med 2012; 186: 155-161.

18 Abellan van Kan G, Rolland Y, Bergman H, et al. The I.A.N.A Task Force on frailty assessment of older people in clinical practice. J Nutr Health Aging 2008; 12: 29-37.

19 Hollman JH, Beckman BA, Brandt RA, et al. Minimum detectable change in gait velocity during acute rehabilitation following hip fracture. J Geriatr Phys Ther 2008; 31: 53-56.

20 van Hedel HJ, Dietz V, Curt A. Assessment of walking speed and distance in subjects with an incomplete spinal cord injury. Neurorehabil Neural Repair 2007; 21: 295-301.

21 Flansbjer UB, Holmback AM, Downham D, et al. Reliability of gait performance tests in men and women with hemiparesis after stroke. J Rehabil Med 2005; 37: 75-82.

22 Perera S, Mody SH, Woodman RC, et al. Meaningful change and responsiveness in common physical performance measures in older adults. J Am Geriatr Soc 2006; 54: 743-749.

23 Onder G, Penninx BW, Lapuerta P, et al. Change in physical performance over time in older women: the Women's Health and Aging Study. J Gerontol A Biol Sci Med Sci 2002; 57: M289-M293.

24 Puhan MA, Mador MJ, Held U, et al. Interpretation of treatment changes in 6-minute walk distance in patients with COPD. Eur Respir J 2008; 32: 637-643. 
Schunemann HJ, Griffith L, Jaeschke R, et al. Evaluation of the minimal important difference for the feeling thermometer and the St. George's Respiratory Questionnaire in patients with chronic airflow obstruction. J Clin Epidemiol 2003; 56: 1170-1176.

26 Guyatt GH, Osoba D, Wu AW, et al. Methods to explain the clinical significance of health status measures. Mayo Clin Proc 2002; 77: 371-383.

27 Revicki D, Hays RD, Cella D, et al. Recommended methods for determining responsiveness and minimally important differences for patient-reported outcomes. J Clin Epidemiol 2008; 61: 102-109.

28 Puhan MA, Chandra D, Mosenifar Z, et al. The minimal important difference of exercise tests in severe COPD. Eur Respir J 2011; 37: 784-790.

29 Hardy SE, Perera S, Roumani YF, et al. Improvement in usual gait speed predicts better survival in older adults. J Am Geriatr Soc 2007; 55: 1727-1734.

30 Shimada H, Suzuki T, Suzukawa M, et al. Performance-based assessments and demand for personal care in older Japanese people: a cross-sectional study. BMJ Open 2013; 3: e002424. 